\title{
Achievable Performance Gain of IEEE 802.11 Multi-rate Link Adaptation Algorithm with Cross-layer Design
}

\author{
Zuoyin Tang, Jianhua He, Yan Zhang, and Zhong Fan
}

\begin{abstract}
Link quality based rate adaptation which aims at link throughput optimization has been used widely for IEEE 802.11 networks. However, from system perspective network performance is affected by not only link quality but also random channel access at the MAC layer. Selection of transmit mode for optimal link throughput can cause performance loss of MAC throughput. In this paper we investigate this issue and propose a generalized cross-layer rate adaptation algorithm which considers jointly link quality at the physical layer and random channel access at the MAC layer to optimize network throughput. The objective is to examine the potential benefits that can be offered by cross-layer design. An efficient analytic model is proposed to evaluate rate adaptation algorithms operated under dynamic channel and multi-user access environments. The proposed rate adaptation algorithm is compared to the link throughput optimization based rate adaptation algorithm. It is found that rata adaptation by optimizing link layer throughput can result in large performance loss at the MAC layer, which can not be compensated by the means of optimizing MAC access mechanism alone. Numerical results show that the cross-layer design can achieve consistent and considerable performance gains of up to $20 \%$. Therefore cross-layer rate adaptation design is promising and deserves to be exploited in practical rate adaptation design for IEEE 802.11 networks.
\end{abstract}

Index Terms-Medium Access Control, Link adaptation, Performance modeling, Wireless Networks, IEEE 802.11, Markov Chain

\section{INTRODUCTION}

IEEE 802.11 wireless local area network (WLAN) physical layers (PHYs) support multiple transmission rates [1]. Link adaptation has been widely used to select transmission rate in an adaptive manner to respond to the dynamic wireless channel conditions [7]. Link adaptation algorithms can be classified into two broad categories: SNR based or packet retransmission based. In the SNR based algorithms, received signal strength (RSS) is used as the indication of link quality. Then transmission mode is selected based on the average or

Zuoyin Tang (e-mail: z.tangleaston.ac.uk) is with School of Engineering and Applied Science, Aston University, UK. Jianhua He (e-mail: j.he@swansea.ac.uk) is with the School of Engineering, Swansea University, UK. Yan Zhang (e-mail: yanzhangesimula.no) is with Simula Research Laboratory and Department of Informatics, University of Oslo, Norway. Zhong Fan (e-mail: Zhong.Fandtoshiba-trel.com) is with Toshiba Telecommunications Research Laboratory, Bristol, UK. instantaneous RSS information from a predetermined SNRrate table to optimize the link throughput [3] [4] [6]. RBAR is a typical example of SNR based algorithms. In the packet retransmission based link adaptation algorithm, the transmitting station counts the outcome (either successful or failed) of each transmission attempt. Based on the packet transmissions (losses) history, the transmitting rate can be adaptively raised by a level or fallback [7] [5] [8] [11] [9].

Basically retransmission based link adaptation algorithms are simple to implement. In the situations where wireless stations contend for channel access, the transmitting station can not distinguish if the cause of packet loss is due to packet corruption or packet collision. The unnecessary selection of lower transmission mode due to packet collision can significantly degrade 802.11 network performance, as reported in [8] [9]. For $802.11 \mathrm{n}$ networks the performance degradation can be more prominent [2]. Therefore SNR table based link adaptation algorithms are preferable for 802.11n networks.

In the traditional SNR table based link adaptation algorithms, the target has been set to purely optimize link throughput based on channel SNR information. We call such algorithms link throughput optimization based rate adaptation (LTRA). However, from the network point of view, optimization of link throughput does not necessarily lead to optimal MAC layer throughput. Obviously packet collision due to competition on the channel access and the distributed coordination function (DCF) at the 802.11 MAC layer will also have large impacts on the MAC layer throughput.

In this paper we are motivated to investigate a cross-layer SNR based link adaptation algorithm. The aim of this work is to optimize the MAC layer throughput, by the design of so-called MAC throughput optimization based rate adaptation (MTRA). The contributions of this paper are mainly in three folds. Firstly the algorithm MTRA is proposed to investigate the performance gain that can be achieved by exploiting crosslayer design for rate adaption. The research findings can help provide insights into future rata adaptation design for 802.11 networks. Secondly an Markov chain based analytical model is developed for SNR based rate adaptation algorithms, in order to efficiently quantize the performance gains of MTRA algorithm. Both rata adaptation for dynamic channel qualities 
and random channel access at the MAC layer are jointly modeled over a channel with finite Markov SNR states and shared by multiple users. Thirdly the analytic model is used for thorough performance evaluation of LTRA, MTRA and fixed rate algorithms. We investigate the impact of channel estimation errors, RTS/CTS access and adaptive control of initial backoff contention window size. The effectiveness of MTRA algorithm is examined. Our initial work on this topic has been reported in [16]. An analytic model has been proposed by the authors for ARF with multiple user access scenarios [12]. But analytic model for SNR based rate adaptation algorithms has not been reported under dynamic channel conditions and multiple user access. To the best of our knowledge, our work on cross-layer design of SNR based rate adaptation algorithm is the first of its kind. In this paper we have a thorough investigation of the analytic model and performance evaluation of LTRA and MTRA algorithms, which can help find out the reason behind the performance gain of MTRA and affirm the effectiveness of cross-layer design for rata adaptation.

The left of the paper is organized as follows. Link adaptation algorithms LTRA and MTRA are presented in Section II. Analytical model for the rate adaptation algorithms are proposed in Section III. System configuration and numerical results are presented and discussed in Section IV and V, respectively. Section VI concludes the paper.

\section{Link AdAptation Algorithms}

As the main purpose of this paper is to investigate the performance gain of exploiting the cross-layer design on link adaptation, we will consider only general algorithms for both LTRA and MTRA. We assume that both transmitting stations and receiving stations have 2 antennas. In the investigated 802.11n PHY, we use the basic 802.11a PHY augmented by 2 by 2 MIMO antennas and space time block coding (STBC) to explore space and temporal diversity gains. The work reported in this paper can be extended to MIMO and spatial multiplexing (SM) to explore multiplexing gains. In the $802.11 \mathrm{a}$ physical layer, there are 8 transmission modes with different modulation and coding configurations for single input single output (SISO) antennas. Each of the 8 basic transmission modes can combined with STBC to get 8 new transmission modes. As in the investigated network scenarios, each transmission mode with STBC always achieves better performance than that with SISO, we will only investigate link adaptation algorithms with STBC.

\section{A. LTRA Algorithm}

For a given channel SNR and corresponding packet error rate (PER), which is denoted by $\left(p_{e r}\right)$, operational transmission mode $r$ is chosen by the LTRA algorithm to optimize the link layer throughput (denoted by $S_{l}$ ). The link throughput $S_{l}$ with
TABLE I

TRANSMISSION MODE $r$ PARAMETERS OF MODULATION, TRANSMISSION RATE $R_{r}$, CODING RATE $C_{r}$ AND EFFECTIVE BIT RATE $B_{r}$.

\begin{tabular}{|l|c|c|c|c|}
\hline Mode & Modulation & $R_{r}(\mathrm{Mbps})$ & $C_{r}$ & $B_{r}(\mathrm{Mbps})$ \\
\hline Mode 1 & BPSK & 12 & $1 / 2$ & 6 \\
\hline Mode 2 & BPSK & 12 & $3 / 4$ & 9 \\
\hline Mode 3 & QPSK & 24 & $1 / 2$ & 12 \\
\hline Mode 4 & QPSK & 24 & $3 / 4$ & 18 \\
\hline Mode 5 & 16QAM & 48 & $1 / 2$ & 24 \\
\hline Mode 6 & 16QAM & 48 & $3 / 4$ & 36 \\
\hline Mode 7 & 64QAM & 72 & $3 / 4$ & 54 \\
\hline Mode 8 & 64QAM & 72 & $2 / 3$ & 48 \\
\hline
\end{tabular}

unlimited retransmissions at a specific transmission mode $r$ can be approximated by:

$$
S_{l}=R_{r}\left(1-p_{e r}\right)
$$

where $R_{r}$ is bit rate (bps) at transmission mode $r$. For both LTRA and MTRA algorithms, we assume that the transmitting stations have the information on relationship of PER to SNR, which can be obtained by either theoretic analysis, simulation or field-trial tests. In this paper such relationship of PER to SNR is obtained by simulations.

To determine which transmission mode to use under the SNR, the LTRA simply takes the following procedures:

1) For a range of $S N R$ values and candidate transmission modes, determine the PER and calculate the achievable link throughput $S_{l}$ from (1).

2) Build a SNR-rate table which associates transmission modes with SNR ranges by which the highest link throughput for LTRA.

3) For each instantaneous link SNR value, find the corresponding SNR value in the SNR-rate table and choose the associated transmission mode as the operational transmission mode.

As there can different packet lengths, multiple SNR-rate tables are normally generated and stored in practice.

It is noted that only packet corruption is taken into account for LTRA algorithm, while packet collisions will not affect the operation of LTRA algorithm. However, the MAC layer performance of the $802.11 \mathrm{n}$ networks will be affected by both packet corruption and packet collisions. The MAC layer performance of LTRA algorithm is analyzed by the model presented in Section III.

\section{B. MTRA Algorithm}

In the MTRA algorithm, the transmission mode is chosen for a given link SNR to optimize the MAC layer throughput $S_{m}$. In this algorithm, both packet corruption and packet collisions are taken into account. As packet collision is dependent on the number of wireless stations competing the channel access, the MLTA algorithm needs to calculate $S_{m}$ and find 
the transmission mode which can achieve the highest $S_{m}$ for each SNR values and the number of competing wireless stations. Performance of 802.11 networks operating with fixed transmission mode and single channel SNR can be obtained with the Markov model in [13] [14].

Selection of the best transmission mode in MTRA algorithm is relatively more complex than LTRA algorithm. The procedures for the MTRA algorithm are summarized as below.

1) Obtain performances of $p_{e r}, S_{l}$ versus $S N R$ for each transmission mode by simulation or theoretic analysis.

2) Use the analytic model presented in Section III to calculate MAC layer throughput $S_{m}$ with a fixed $S N R$, a fixed number of wireless stations for each possible transmission mode.

3) Find the best transmission mode achieving the highest MAC layer throughput for the given $S N R$ and the number of wireless stations.

4) Build a table for the best transmission modes associated with the SNR ranges and the number of stations for MTRA algorithm.

5) For each instantaneous SNR value and a given number of stations, choose the associated transmission mode in the pre-defined table for packet transmission.

The above design of MTRA algorithm is general and serves to understand how much performance gain can be obtained by exploiting the cross-layer design. It may not be used as a practical link adaptation algorithm without modification as the number of competing stations and their traffic can be dynamically changing.

Fig. 1 presents the an example relationship of PER to $S_{l}$ for the 802.11a augmented with $2 \times 2$ antennas and STBC for the 8 transmission modes. Fig. 2 shows the corresponding link throughput calculated by (1) for the transmission modes. The best transmission mode for a SNR range for LTRA algorithm can be found from Fig. 2. Table II shows the best transmission modes and their corresponding lower and upper bound SNR thresholds.

TABLE II

TRANSMISSION MODE $r$ FOR LTRA.

\begin{tabular}{|l|c|c|c|c|c|c|}
\hline SNR $(\mathrm{dB})$ & $<0$ & {$\left[\begin{array}{ll}0 & 2.5\end{array}\right)$} & {$\left[\begin{array}{ll}2.5 & 6)\end{array}\right.$} & {$\left[\begin{array}{ll}6 & 8\end{array}\right)$} & {$\left[\begin{array}{ll}8 & 14\end{array}\right)$} & $>14$ \\
\hline Tx mode $r$ & 1 & 3 & 4 & 5 & 6 & 7 \\
\hline
\end{tabular}

\section{Analytical Model}

In this section we will present a Markov-chain based analytical model for both LTRA and MTRA algorithms operating with the basic distributed coordination function (DCF) access scheme. It is trivial to extend the analytical model to the algorithms with RTS/CTS schemes. We assume a single hop wireless LAN where $N$ stations are identical to each other. Each station has saturated traffic to transmit to one

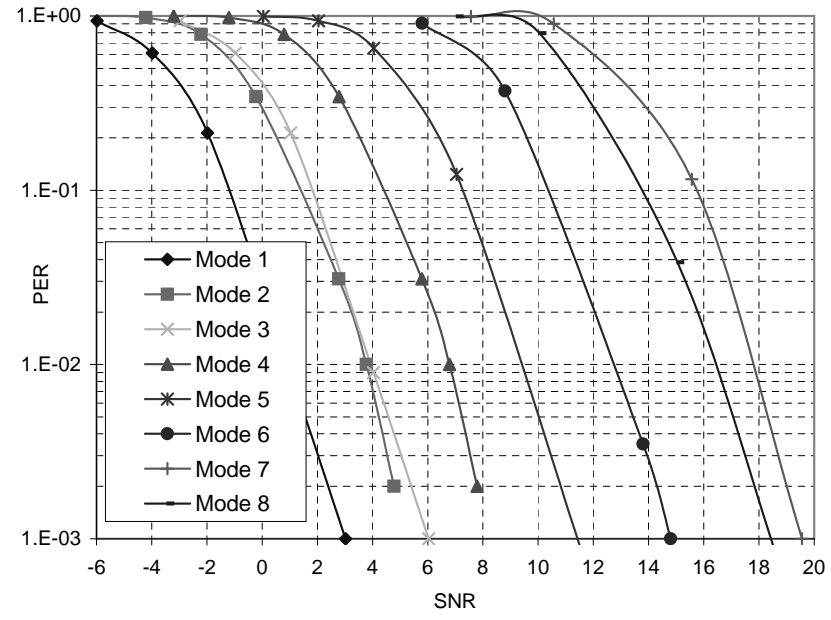

Fig. 1. Packet error rate performances of 802.11 a with $2 \times 2$ STBC.

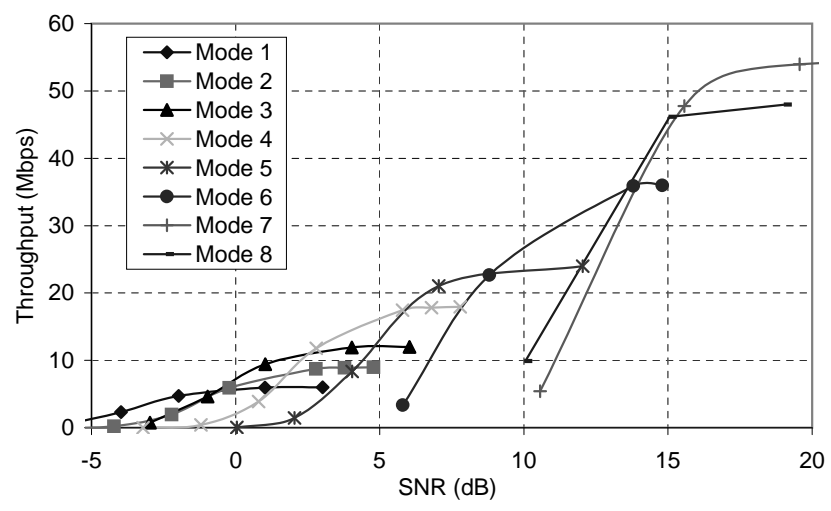

Fig. 2. Link layer throughput of 802.11a with $2 \times 2$ STBC.

of its neighbors. System time is slotted with each time slot of $\delta$ second. We assume that the dynamic channel can be characterized by $N_{n}$ discrete Markov states $S N_{n}, n \in[1, n]$. The SNR states $S N_{n}$ are labeled such that the associated data rate $R_{n}$ are in the non-decreasing order, $n \in[1, n]$. Let $p_{s}$ shown in (2) denote the transition probabilities among the SNR states, where $p_{s}(i, j)$ denotes the transition probability between $S N_{i}$ and $S N_{j}, i, j \in\left[1, N_{n}\right]$.

$$
P=\left[\begin{array}{cccc}
p_{s}(1,1) & p_{s}(1,2) & \ldots & p_{s}\left(1, N_{n}\right) \\
p_{s}(2,1) & p_{s}(2,2) & \ldots & p_{s}(2, n) \\
: & : & \ldots & : \\
p_{s}\left(N_{n}, 1\right) & p_{s}\left(N_{n}, 2\right) & \ldots & p_{s}\left(N_{n}, N_{n}\right)
\end{array}\right]
$$

Let $N_{n}$ denote the number of transmission modes associated with the $N_{n}$ SNR states. Note that the optimal transmission mode for a SNR state has already been selected in terms of link throughput for the LTRA algorithm, but will need to be searched for the MTRA algorithm with the assistance of the proposed analytic model. Let $p_{c o r r}(i, j)$ denote the probabilities that a data packet is corrupted under the $j$ th SNR state with the $i$ th transmission mode, $i, j \in\left[1, N_{n}\right]$ without any interference from other competing stations. Denote $T_{a v g}$ as the average duration of a generalized time slot. 
We assume that each node will transmit with probability of $\tau$ independently to its neighbors at each virtual time slot, and $\tau_{r}$ with transmission rate of $R_{r}$ bps, $r \in\left[1, N_{n}\right]$. We have $\tau=\sum_{r=1} N_{n} \tau_{r}$. Assume that data packet and ACK packet have fixed length of $L_{d t}$ and $L_{a c k}$ bits. Transmissions of a data packet and an ACK packet at transmission rate of $R_{r}$ will last for $T_{d t}(r)$ and $T_{a c k}(r)$ time slots: $T_{d t}(r)=L_{d t} /\left(R_{r} T_{a v g}\right)$ and $T_{a c k}(r)=L_{a c k} /\left(R_{r} T_{a v g}\right)$.

\section{A. Markov Model}

Let $p(i)$ denote the probability of a general time slot being idle for a state operating with the $i$ th transmission mode. We get the probability $p_{c}(i, j)$ that a data packet transmitted with transmission mode $i$ can not be successfully acknowledged due to either packet collision or packet corruption or both under the $j$ th SNR states, $p_{c}(i, j)=1-\left[1-p_{\text {corr }}(i, j)\right][1-p(i)]$, $i, j \in\left[1, N_{n}\right]$.

We can produce a 3-dimension Markov chain for the DCF backoff process of a wireless station with an example shown in Fig.3. For illustration purpose, only 2 SNR states are shown in the figure. For a general Markov state $(r, i, j), r$ represents the order of the transmission rate $R_{r} ; i$ represents the backoff state, meaning that the backoff process is in either the first transmission $(i=0)$ or retransmission attempt $(0<i \leq m)$; $j$ represents the value of the backoff counter, $j \in\left[0, W_{i}-1\right]$. $W_{i}$ is the contention window, $W_{i}=2{ }^{i} W_{0}(i \in[0, m]), W_{m}$ is the maximum contention window, and $m$ is the maximum number of allowed retransmission.

Let $P\left(r_{1}, i_{1}, j_{1} \mid r_{0}, i_{0}, j_{0}\right)$ denote the transition probability of Markov state $\left(r_{0}, i_{0}, j_{0}\right)$ to $\left(r_{1}, i_{1}, j_{1}\right)$. According to the three major types of state transition events, we can obtain the formula for the transition probabilities as below.

- Backoff state $(i, j, k)$ transits to itself with probability of $p_{i}$ due to channel sensed busy, or transit to state $(i, j, k-$ 1 ) if the channel is sensed idle in the beginning of the next time slot, for $k>0$. The stations with backoff counter $k$ becoming 0 will transmit the the head packet in the transmission buffer.

$$
\begin{cases}P\left\{s_{2}, j, k \mid s_{1}, j, k-1\right\} & =p_{s_{1}} \\ P\left\{s_{2}, j, k \mid s_{1}, j, k\right\} & =1-p_{s_{1}}\end{cases}
$$

where $s_{1}, s_{2} \in\left[1, N_{n}\right], j \in[0, m], k \in\left[0, W_{j}\right]$.

- Transmission state $\left(s_{1}, j, 0\right)$ transits to $\left(s_{2}, j+1, k\right)$ with probability of $p_{s}\left(s_{1}, s_{2}\right) p_{c}\left(s_{1}, s_{2}\right) / W_{j+1}$ due to a unsuccessful transmission, or transits to $\left(s_{2}, 1,0\right)$ with probability of $p_{s}\left(s_{1}, s_{2}\right)\left(1-p_{c}\left(s_{1}, s_{2}\right)\right)$ due to a successful transmission, where $\left.s_{1}, s_{2} \in\left[1, N_{n}\right]\right], j \in[0, m-1]$, and $k \in\left[0, W_{0}-1\right]$.

$\left\{\begin{array}{l}P\left\{s_{2}, 0, k \mid s_{1}, j, 0\right\}=p_{s}\left(s_{1}, s_{2}\right)\left(1-p_{c}\left(s_{1}, s_{2}\right)\right) / W_{0} \\ P\left\{s_{2}, j+1, k \mid s_{1}, j, 0\right\}=p_{s}\left(s_{1}, s_{2}\right) p_{c}\left(s_{1}, s_{2}\right) / W_{j+1}\end{array}\right.$ where $s_{1}, s_{2} \in\left[1, N_{n}\right], j \in[0, m-1]$.

- Transmission state $\left(s_{1}, m, 0\right)$ transits to $\left(s_{2}, 0,0\right)$ with probability of $p_{s}\left(s_{1}, s_{2}\right)$ due to the channel SNR state transition, no matter if the transmission is either successful or failed.

$$
P\left\{s_{2}, 0,0 \mid s_{1}, m, 0\right\}=p_{s}\left(s_{1}, s_{2}\right), s_{1}, s_{2} \in\left[1, N_{n}\right]
$$

Denote $b_{r i j}$, as the distributions of states $(r, i, j)$ states, $r \in$ $\left[1, N_{n}\right], i \in[0, m], j \in\left[0, W_{i}-1\right]$. Then we can calculate $b_{r i j}$ using the state transition probabilities (4) and the following condition (6),

$$
\sum_{r=1}^{N_{n}} \sum_{i=0}^{m} \sum_{j=0}^{W_{i}-1} b_{r i j}=1
$$

Transmission probability $\tau$ and $\tau_{r}$ can be approximated by $b_{\text {ri0 }}$,

$$
\left\{\begin{aligned}
\tau & =\sum_{r=1}^{N_{n}} \sum_{i=0}^{m} b_{r i 0} \\
\tau_{r} & =\sum_{i=0}^{m} b_{r i 0}
\end{aligned}\right.
$$

Under the assumption that the channel states sensed by a station is the same as that sensed by the other stations, we can calculate $p$ by (8) if the SNR transition is synchronized for all the stations,

$$
p(r)=1-[1-\tau(r)]^{N-1}
$$

If all the stations undergo fully independent SNR transition, we can calculate $p(r)$ by (9)

$$
p(r)=1-(1-\tau)^{N-1}
$$

It is clear that $p$ is a function of transmission probability $\tau$. Given $p_{s}$ and $p_{\text {corr }}$, with only two unknowns $\tau$ and $p$, numerical methods can then be used to calculate them from (7) and (8) or (9).

\section{B. Throughput Calculation}

Let $S$ denote single node MAC layer throughput, defined as number of upper layer data bits successfully delivered at the MAC layer by a node during a second. It can be calculated as the ratio of payload information successfully transmitted by a node in a time slot to the length of a time slot. Let $p_{i d l e}$ be the probability that the channel is sensed idle by the tagged node in a time slot. Let $p_{\text {err }}(r)$ denote the probability of an event (denoted by $E_{\text {err }}(r)$ ) that an acknowledgement (ACK) packet for a data packet transmission is not correctly received in a time slot and the lowest data rate used for the packet(s) transmitted in the time slot is $R_{r}$. The reason an ACK packet is not received for a data packet maybe packet 


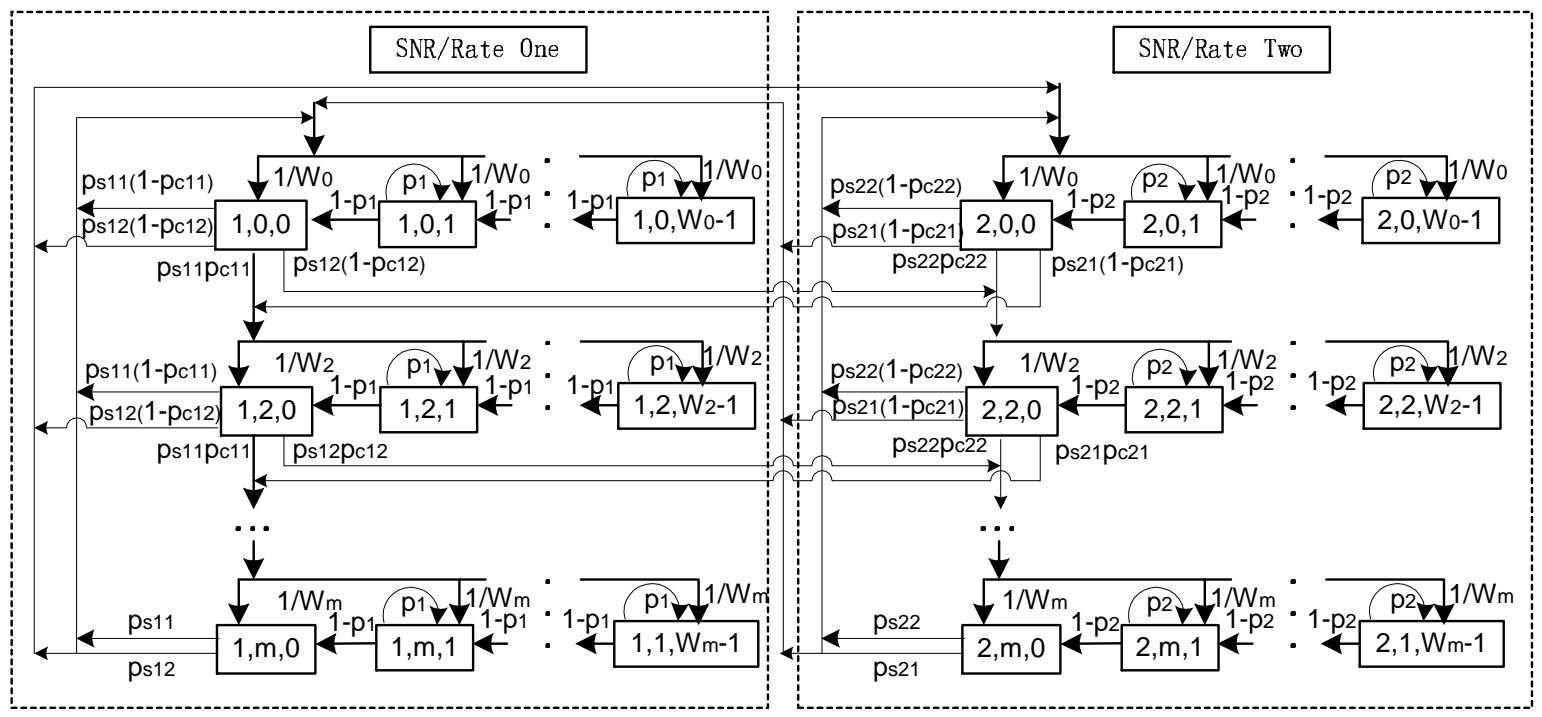

Fig. 3. Markov chain for the backoff procedure of a station operating with two transmission rates.

corruption or collision of data or ACK packets. Let $p_{s u c}(r)$ denote the probability of an event (denoted by $E_{\text {suc }}(r)$ ) that an ACK packet for a packet transmission is successfully received in a time slot and the data rate used for the packet transmission is $R_{r}$.

Let $T_{\text {err }}(r)\left(T_{\text {suc }}(r)\right)$ denote the average duration of a time slot during which an event $E_{\text {err }}(r)\left(E_{\text {suc }}(r)\right)$ happened. Then $T_{\text {err }}(r)$ and $T_{\text {suc }}(r)$ can be expressed for the basic access scheme as below:

$$
\left\{\begin{array}{l}
T_{\text {suc }}(r)=D I F S+S I F S+\left(L_{d}+L_{a}\right) / R_{r} \\
T_{\text {err }}(r)=D I F S+L_{d} / R_{r}+\text { EIFS }
\end{array}\right.
$$

where SIFS and EIFS are shortest inter-frame space and extended inter-frame space, defined by IEEE 802.11 standard for different types of channel access.

We can also calculate the defined probabilities of $p_{i d l e}$, $p_{\text {suc }}(r)$ and $p_{\text {err }}(r)$ as follows:

$$
\left\{\begin{array}{l}
p_{\text {idle }}=(1-\tau)^{(N)} \\
p_{\text {suc }}(r)=N \tau_{r}\left(1-p_{\text {er }}\right)\left(1-\sum_{j=1}^{r} \tau_{r}\right)^{(N-1)} \\
p_{\text {err }}(r)=\sum_{i=2}^{N}\left(1-\sum_{j=1}^{r} \tau_{r}\right)^{i}+N \tau_{r} p_{e r}\left(1-\sum_{j=1}^{r} \tau_{r}\right)^{(N-1)}+ \\
N \tau_{r}\left(1-p_{e r}\right)\left[\left(1-\sum_{j=1}^{r} \tau_{r}\right)^{(N-1)}-(1-\tau)^{(N-1)}\right]
\end{array}\right.
$$

Then the average duration of a time slot $T_{a v g}$ and single node MAC layer throughput $S$ can be calculated by (12):

$$
\left\{\begin{array}{l}
T_{a v g}=p_{i d l e} \delta+\sum_{r=1}^{N_{n}}\left[p_{\text {err }}(r) T_{\text {err }}(r)+p_{\text {suc }}(r) T_{s u c}(r)\right] \\
S=\frac{L_{d} \sum_{r=1}^{N_{n}} \tau_{r}\left(1-p_{\text {cr }}\right) C_{r}}{T_{\text {avg }}}
\end{array}\right.
$$

\section{NumeriCAL RESUlts}

In this Section, we present some typical analytical results of both LTRA and MTRA algorithms. For performance comparison purpose, the results for a fixed rate algorithm are also included, The fixed rate algorithm simply uses a fixed transmission mode for all packet transmissions. In the studied one-hop wireless LAN, the number of stations is fixed and configured to 5, 15, 25 and 35. All the stations are identical with saturated traffic and operating with the same link adaptation algorithm (LTRA, MTRA or fixed rate algorithm). There are three sets of channel SNR states, with

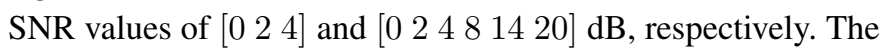
associated transmission modes chosen by LTRA for a given SNR are listed in Table. II. For simplicity, we set the SNR state transition matrix $p_{s}(i, j)$ as below:

$$
\begin{cases}p_{s}(i, i)=p_{s s}, & i \in[1,6] \\ p_{s}(i, j)=\left(1-p_{s s}\right) / 5, & i, j \in[1,6], i \neq j .\end{cases}
$$

In (13), $p_{s s}$ is a configurable variable and denote the probability of a SNR state transiting to itself. It is fixed and set to $\left[\begin{array}{llll}0.2 & 0.45 & 0.7 & 0.95\end{array}\right]$ in our analysis respectively.

An example SNR state transition sequence with $N_{n}=6$ and $p_{s s}=0.7$ is presented in Fig. 4 .

Fig. 5 to Fig. 6 present the aggregate MAC layer throughput obtained by LTRA, MTRA and fixed rate algorithms versus number of stations with packet length of 3000 bits and 5000 bits, respectively. Fig. 5 (a), (b) and (c) correspond to SNR

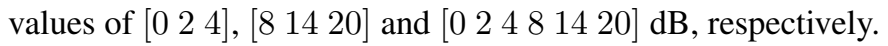
Both MTRA and LTRA outperform fixed rate algorithm for all the investigated scenarios.

(12) It is also observed from the figures that the performance of the MTRA algorithm is consistently better than that of the LTRA algorithm by up to $20 \%$. The performance gains are more prominent with large packet size and large number 


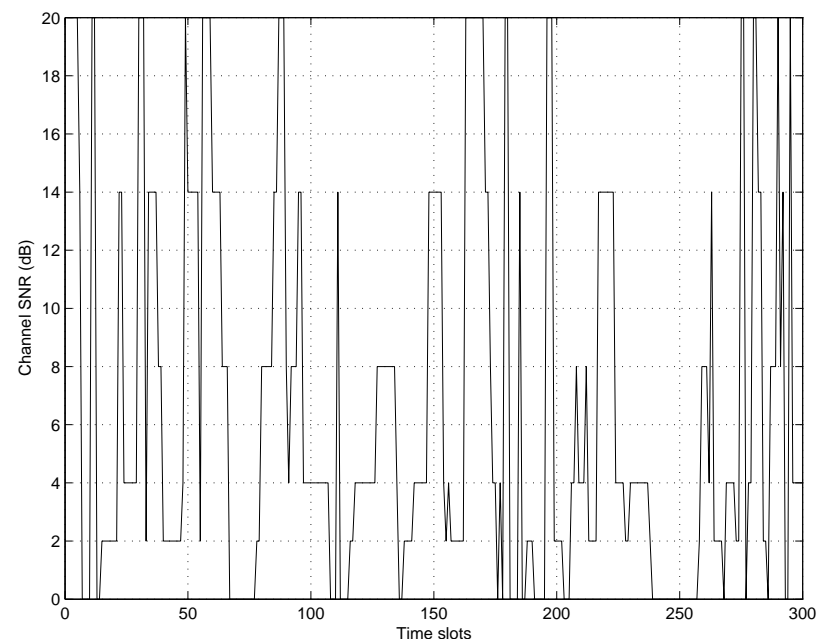

Fig. 4. SNR Markov state transition sequences with $N_{n}=6$ and $p_{s s}=0.7$.

of competing stations. It demonstrated that the cross-layer approach used in the MTRA algorithm can bring considerable performance gain and should be exploited in the design of practical link adaptation algorithms for 802.11n networks.

\section{CONCLUSIONS}

In this paper, we proposed and investigated a generalized cross-layer SNR based rate adaptation algorithm MTRA for IEEE 802.11 networks. Both link quality at the PHY layer and channel access competition at the MAC layer are exploited to select the best transmit mode in terms of optimal MAC throughput. The cross-layer rate adaptation is different from the widely used link adaptation algorithm LTRA which aims to optimize link throughput. We proposed an Markov chain based analytic model to evaluate the performance gains of MTRA algorithm over LTRA algorithm. The impact of channel SNR dynamics, rate adaptation and channel access schemes on MAC throughput is taken into account. The results show that cross-layer rate adaptation design in MTRA algorithm can bring considerable MAC throughput improvement by up to $20 \%$. Through the in-depth investigation on the performance improvement by MTRA algorithm, it was found that the improvement comes mainly from link quality adaptation rather than channel congestion adaptation. Selection of transmit mode by optimizing link throughput in LTRA algorithm can result in large performance loss at the MAC layer. And such performance loss can not be compensated by the means of optimizing access mechanisms at the MAC layer alone (such as RTS/CTS and adaptive contention window control). Therefore cross-layer design in the MTRA algorithm is quite effective and deserves to be exploited in design of practical rate adaptation algorithms for emerging advanced IEEE 802.11 networks.

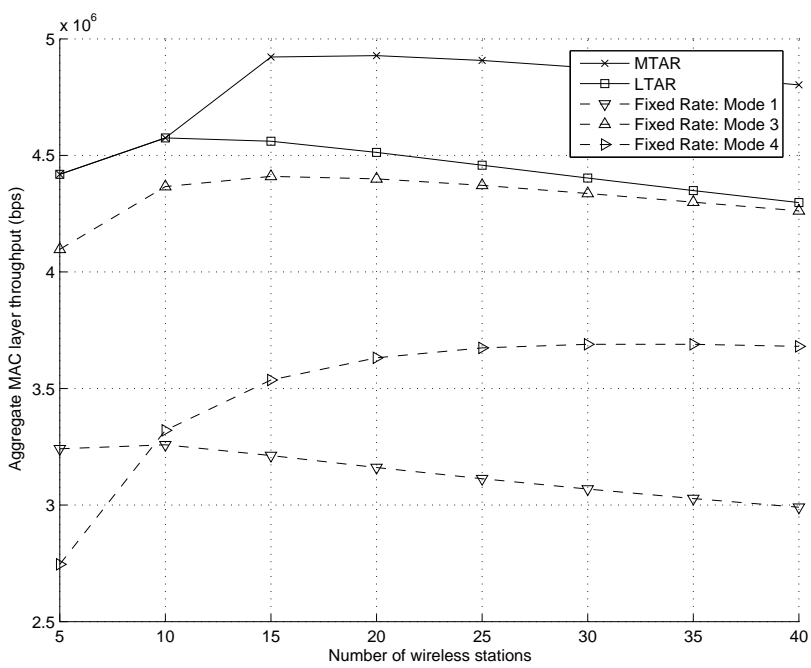

(a) $\mathrm{SN}=(0,2,4) \mathrm{dB}$.

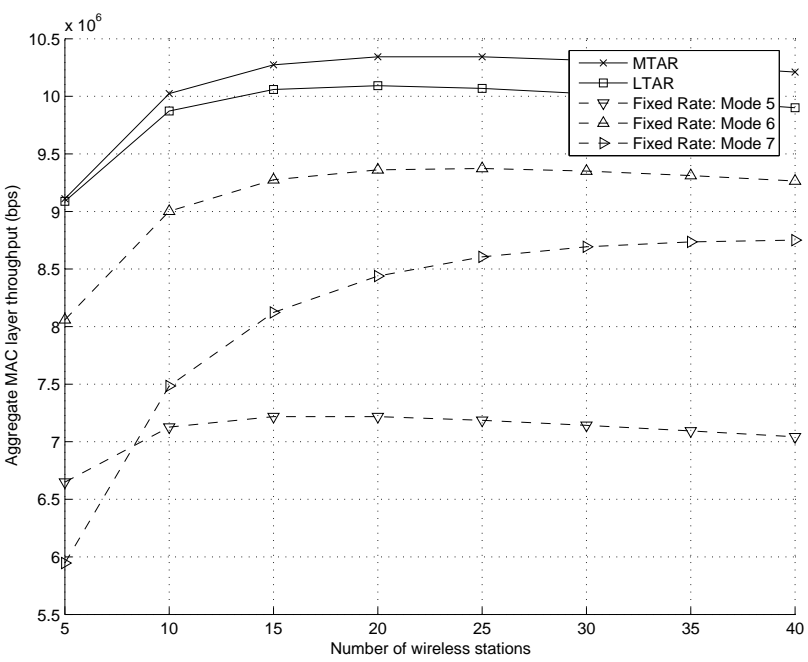

(b) $\mathrm{SN}=(8,14,20) \mathrm{dB}$

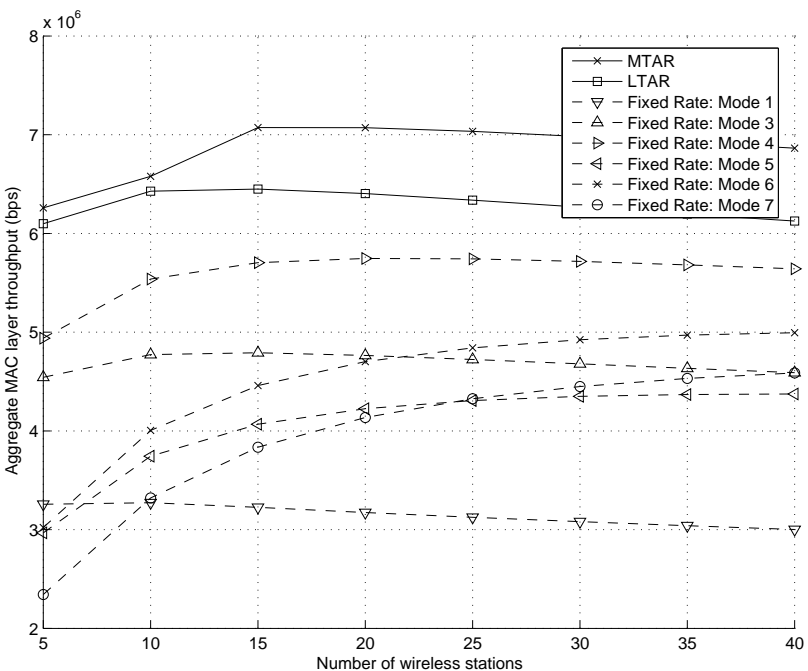

(c) $\mathrm{SN}=(0,2,4,8,14,20) \mathrm{dB}$.

Fig. 5. Aggregate MAC layer throughput versus number of stations with data packet length $L_{d t}=3000$ bits. 


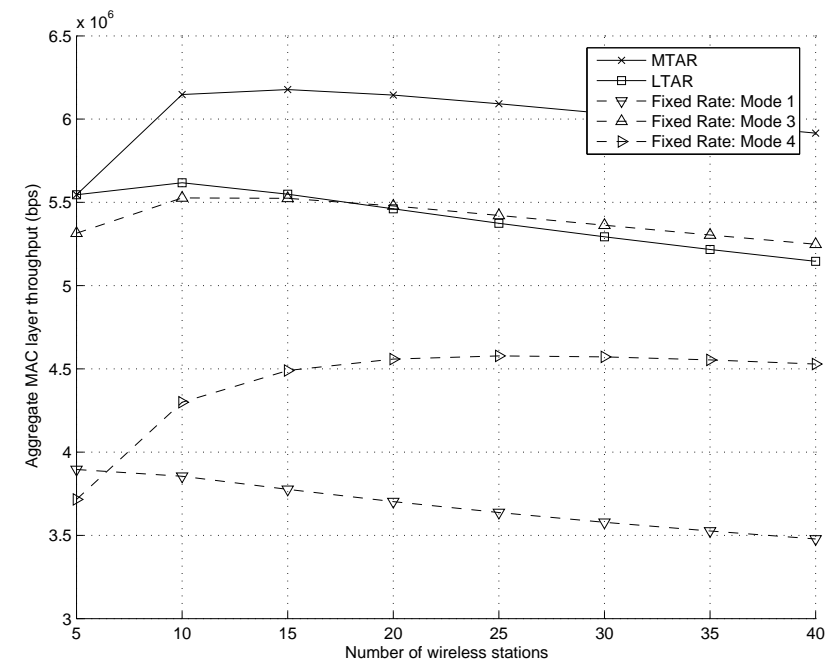

(a) $\mathrm{SN}=(0,2,4) \mathrm{dB}$.

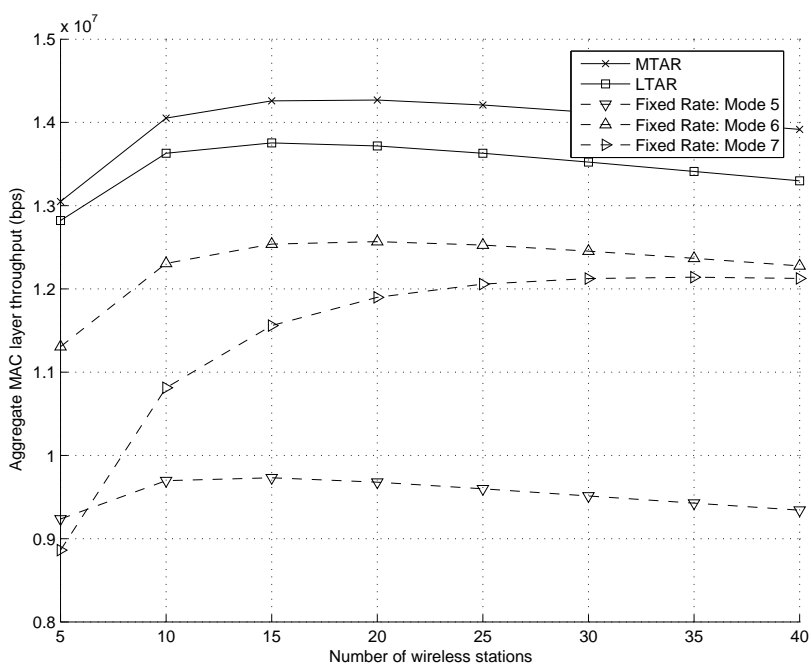

(b) $\mathrm{SN}=(8,14,20) \mathrm{dB}$.

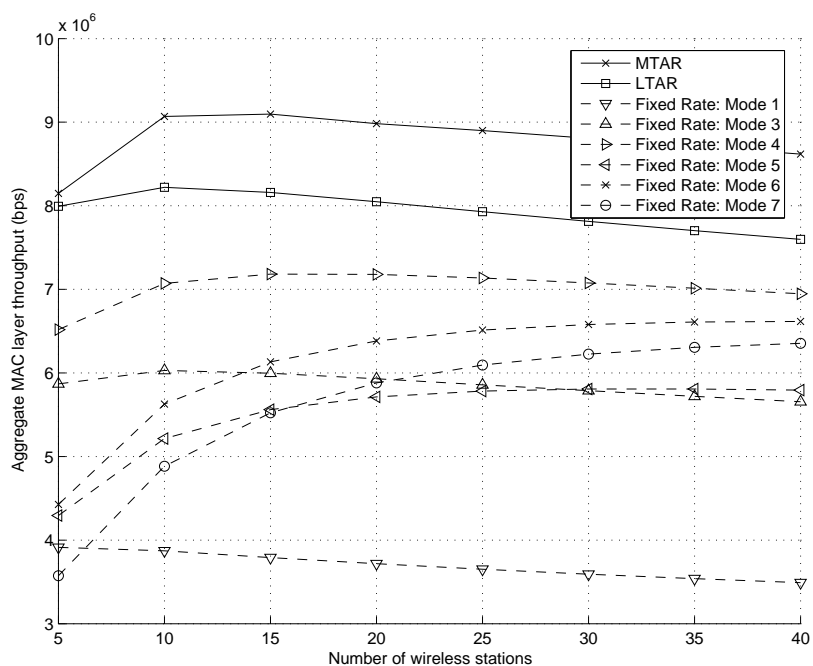

(c) $\mathrm{SN}=(0,2,4,8,14,20) \mathrm{dB}$.

Fig. 6. Aggregate MAC layer throughput versus number of stations with data packet length $L_{d t}=5000$ bits.

\section{ACKNOWLEDGEMENT}

The authors would like to thank all the anonymous reviewers for their constructive comments which help improve the quality of this paper. The work of Dr Jianhua He was supported partly by the UK Engineering and Physical Sciences Research Council (EPSRC) with grant reference number EP/1010157/1.

\section{REFERENCES}

[1] IEEE standard for wireless lan medium access control (MAC) and physical layer (PHY) specifications, 1997.

[2] IEEE 802.11 standard, Amendment 5: Enhancements for Higher Throughput. 2009.

[3] J. Pavon, S. Choi, Link Adaptation Strategy for IEEE 802.11 WLAN via Received Signal Strength Measurement, Proc. of IEEE ICC'03, 2003.

[4] Daji Qiao and Sunghyun Choi, Goodput Enhancement of IEEE 802.11a Wireless LAN via Link Adaptation, in Proc. IEEE ICC'03, pp. 19952000, June 2001.

[5] J. Wu, Hunh-Huan Liu and Yi-Jen Lung, An Adaptive Multirate IEEE 802.11 Wireless LAN. in Proc. ICIN'01, pp. 411-418, 2001

[6] G. Holland, N. Vaidya, and P. Bahl. A rate-adaptive MAC protocol for multi-hop wireless networks. In Proc. ACM/IEEE MobiCom, July 2001

[7] A. Kamerman and L. Monteban. WaveLAN-II: A high-performance wireless lan for the unlicensed band. ATT Bell Laboratories Technical Journal, pages 118-133, 1997.

[8] Mathieu Lacage, Hossein Manshaei, and Thierry Turletti. IEEE 802.11 rate adaptation: A practical approach. In Proc. of MSWiM'04, 2004.

[9] John C. Bicket, Bit-rate Selection in Wireless Networks. Thesis of Master, MIT, Feburary 2005.

[10] Eddie Kohler, et al, The Click modular router. ACM Trans. on Computer Systems, 18(4), November 2000.

[11] Madwifi. http://sourceforge.net/projects/madwifi.

[12] Jianhua He, et al, Modeling Link Adaptation Algorithm for IEEE 802.11 Wireless LAN Networks. in Proc. of ISWCS, September, 2006.

[13] G. Bianchi. Performance analysis of the IEEE 802.11 distributed coordination function. IEEE JSAC, 18:535-547, March 2000.

[14] J. He, L. Zheng, Z. Yang, and C. Chou. Performance analysis and service differentiation in IEEE 802.11e EDCF. In Proc. of $L C N$, pages 184-190, October 2003.

[15] J. He, L. Zheng, Z. Yang, and C. Chou. Investigation of service differentiation mechanisms for IEEE 802.11 wireless LAN. IEICE Tran. on Commun., June 2004.

[16] Z. Tang, J. He, Y. Zhang, Z. Fan. Investigation of cross-layer link adaptation algorithm for IEEE 802.11n Networks. In Proc. of IWCMC, 2010 . 